\title{
Design and Performance analysis of hybrid energy harvesting and WSN application for more life time and high throughput
}

\author{
${ }^{1}$ Shiva Kumar V., ${ }^{2}$ Rajashree V. Biradar, ${ }^{3}$ V. C. Patil \\ ${ }^{1}$ Dept. of CSE,RYMEC, Ballari, Karnataka, India, \\ ${ }^{2}$ Dept. of CSE, BITM, Ballari,Karnataka, India \\ ${ }^{3}$ Dept. of ECE Ballari,Karnataka, India \\ 1'shiva.vadla@gmail.com, ${ }^{2}$ rajashreebiradar@yahoo.com and ${ }^{3}$ patilvc@rediffmail.com
}

Received: June 29, 2021. Revised: December 8, 2021. Accepted: January 16, 2022. Published: January 17, 2022.

Abstract - the technology of wireless sensor-actuator networks (WSANs) is widely employed in the applications of IoT due to its wireless nature and it does not involve any wired structure. The wireless systems that are batterydriven can easily reconfigure the existing devices and sensors efficiently in the manufacturing units without employing any cable for power operation as well as for communication. The wireless sensor-actuator networks that are based on IEEE 802.15.4 consumes significantly less power. These networks are designed and built costeffectively by considering the capacity of battery and expense so that they can be employed for many applications. The application of a typical wireless Autonomous Scheduling and Distributed Graph Routing (DDSR) has illustrated the reliability of employing its basic approaches for almost ten years and it consists of the accurate plot for routing and time-slotted channel hopping therefore ensuring accurate low-power wireless communication in the processing site. Officially declared by the controversial statements associated with the government of Greek experiences fourth industrialization. There is a huge requirement for sensor nodes link via WSAN in the industrial site. Also, reduced computational complexity is one of the drawbacks faced by the existing standards of WSAN which is caused because of their highly centralized traffic management systems and thereby significantly improves the consistency and accessibility of network operations at the expense of optimization. This research work enables the study of efficient Wireless DGR network management and also introduces an alternative for DDSR by enabling the sensor nodes to determine their data traffic routes for the transmission of data. When compared to the above two physical routing protocols, the proposed technique can drastically improve the performance of a network, throughput, and energy consumption under various aspects. Energy harvesting (EH) plays a significant role in the implementation of large IoT devices. The requirement for subsequent employment of power sources is eliminated by the efficient approach of Energy Harvesting and thereby providing a relatively close- perpetual working environment for the network. The structural concept of routing protocols that are designed for the IoT applications which are based on the wireless sensor has been transformed into "energyharvesting-aware" from the concept of "energy-aware" because of the development in the Energy harvesting techniques. The main objective of the research work is to propose a routing protocol that is energy-harvestingaware for the various network of IoT in case of acoustic sources of energy. A novel algorithm for routing called Autonomous Scheduling and Distributed Graph Routing (DDSR) has been developed and significantly improved by incorporating a new "energy back-off" factor. The proposed algorithm when integrated with various techniques of energy harvesting enhances the longevity of nodes, quality of service of a network under increased differential traffic, and factors influencing the accessibility of energy. The research work analyses the performance of the system for various constraints of energy harvesting. When compared to previous routing protocols the proposed algorithm achieves very good energy efficiency in the network of distributed IoT by fulfilling the requirements of QoS.

Keywords-: Routing Algorithm, WSN, Wireless network, DDSR, QoS, IoT, WSAN, DGR and Scheduling algorithm. 


\section{INTRODUCTION}

$\mathrm{D}$ evelopment in the technology of senor has paved the way for the design of low powered and relatively small, sensors that are well furnished with programming ability, efficiency in detecting various parameters, and competency in communication that are wireless. Since the sensor technology is cost-effective, the network incorporates several hundreds of sensors and thus improving the efficiency, area availability, and data precision. In obscure and undeveloped areas, the networks of the wireless sensor provide necessary information or data regarding common ecological factors, remote systems, and so on. When compared to wired communication, the network of wireless sensor offers many advantages like simplicity in designing a network (minimizing initial cost overhead), high speed ( a network with relatively small sensors can be allocated over a wide area), fault tolerance ( malfunctioning of one node do not impact on the network functioning), self-oriented (the reconfiguring ability of node itself) and some of the intrinsic problems faced by wireless sensors are limited bandwidth, data transmission that is errorfree, interference-free and so on. Since cell-phones are the most widely used wireless nodes they use only specific batteries to draw the energy and do not require any constant supply of power. Therefore, this reduces the total energy accessible to the nodes. Furthermore, these wireless nodes find it hard to replace both the sensor nodes and battery packs in few areas, therefore, it is essential to maximize the durability of networks by placing a set of new nodes that can recharge the entire area [1]. A pre-defined implementation is required to identify the nodes that are not working and preferentially substitute them by reducing a few network benefits. An optimum sensor system must possess location responsiveness and addressing that is based on the attribute. One more essential aspect of sensors is that it should respond instantly to significant environmental variations such as an application that are based on time. The receiver must be given information regarding other remaining nodes that possess small delay and thus ensuring efficient utilization of bandwidth in the wireless media. As a result, data-centric protocols that have data accumulation efficiency, consistently allocating power dissipation, reduced energy to maintain network durability, and eliminating the constraint of a single node (excluding BS) are essential for the wireless sensor networks. As discussed in the previous paper [4] the conventional network protocols are not applicable for wireless communication that is described for MANETs. Recently, a data transmission protocol which is energy efficient named LEACH has been presented [2], and based on the data obtained by BS, the hierarchical clustering is achieved. However, to minimize energy, the cluster-head $(\mathrm{CH})$ and many nodes are frequently varied by the BS. The cluster head receives data from the sensors, analyses, and then transmits to BS. The consumption of energy is evenly allocated by arbitrarily rotating the $\mathrm{CH}$ if not the cluster head that is nearer to BS will not allow data to be transmitted and power dissipation compels them to perish quickly when compared to other nodes. Constant re-clustering is done by the BS to allow another active node to function as cluster head when one of the $\mathrm{CH}$ because for some reason is unable to interact with its node members or with the BS. The information regarding how a node is established and on what basis the cluster head must be chosen is addressed in [3].

The common drawback noticed was how to resolve the queries of the users and in what way the required data is routed. The majority of the existing protocols acknowledge that a sensor collects the information regularly from the system and when a query arrives the protocol reacts to it. In LEACH [2] the cluster head receives the information continuously, and after the process of clustering, the $\mathrm{BS}$ receives the information to store the data. In sensor networks, particularly the applications which are based on time are not focused specifically. The sensor networks must specify the final users to dynamically deal with energy proficiency, precision, and time taken for responding. In this work, we mainly aim at advancing a routing protocol that is efficient and a detailed request managing process that satisfies the above requirements.

The major issue faced in decentralized IoT is the propagation of cost-effective data which is illustrated by many research work [6]. Several research works have been accomplished in the area of data network aggregation [7], without any compression loss and with compression loss [8] (the main concern is to improve the efficiency of energy by minimizing the total bits to be transmitted), as well as enhancement of various objects present in the wireless communication [9]. Particularly, the specific level of compression, quality of signal trading, and quality of service are allowable for a long-lasting network in several applications of the internet of things. In this research work, we visualize the networks of IoT that inevitably switch their activity to various sources, their positions, rate of transmission (i.e. encoding of source), distribution flow systems, and quality of service required by the application. Therefore, we propose the application to overcome the drawbacks of distributed learning techniques, reviewing source compression, and distribution flow issues. In general, we collectively describe the issues related to lossy data compression at the input side and a successful routing path is established towards the data distribution center of the Internet of things (the gateway of the Internet of things is also known as a sink). This helps in analyzing the basic difference between the efficiency of the distortion rate at the inputs and outlay required for transmitting the necessary data and addressing the issues of distribution flow. The primary objective is to interpret exactly how much computation has to be carried out near the input with the help of a few lossy data compression algorithms. Thus the compressed data is processed successfully and communicated via a plot of a network by collectively handling compression and routing. Make sure that the compression is intrinsically combined with the efficiency of interconnected network constraints, their destinations, total inputs and their locations, and the communication node's potency. While identifying the drawbacks, we arrived at the concept of possessing transmission overheads in contrast to the interference of signal and thereby enabling the study of an ideal cost-distortion area (allocative efficiency) of a network. 
The main difference between the existing and the proposed work is that no hard-coded protocol standards are used to ensure the quality of source compression and process the data collected. However, these responses generally arrive as alternatives for the process of distributed learning which contains the iterative transmission process among the local signals and the nodes [5]. The nodes do not possess any information regarding the entire network process or status of network standardization, and also they have no idea regarding the detailed structure of the network. Instead, local communications enable the design of a distributed system to integrate the process of optimization worldwide. An initial data centralized system is introduced in the research work of [10]. Several research works have been carried out in the field of distributed WSNs namely source encoding, forwarding, and enhancement techniques for multi-objective. Few research works are concerned with encoding of data at the input [11], and some works enable the accumulation of data at the arbitrator nodes while forwarding the data to the destination node. The paths for routing can be predetermined by the advanced computation [12] or determined with the help of a functional approximation process that requires accumulation and data forwarding network [11]. Since the system implements encoding at the input, the researchers of are concerned with combined data encoding and collection of data by consuming the energy computed for data encryption in contrast to the energy utilized for the communication. The strategy discussed above is analogous to the proposed one and the primary distinction is that the paths for routing and encoding standards at the inputs are simultaneously determined and are executed as the process of deep learning. The drawbacks of combined routing and encoding are introduced by employing the theory of Lyapunov optimization in [13]. On the other hand, the path for routing is already defined and is not implemented with the encoding procedures. The research work of explores a conceptual framework for data collection networks along with data encoding techniques, where every individual node pre-processes the data collected before delivering the information to the local network. The proposed work mainly concentrates on the cost-effective encoding and transmission scheduling for the network with a single hop and considering encoding and communication expenses under a rescheduling limit.

Many research works utilizes spatial correlation for data accumulation example [13]. These works mainly concentrate on the information generated from the temporally correlated inputs and forwards the same information to the destination. Encoding inevitably leads to in-network accumulation, and researches are conducted to explore the difference between routing and accumulation. The issue of routing was not considered by the analysis of the distributed method for compressive sensing, for example, illustrated in [10]. For the multi-objective enhancement, there exist several research works. For example, [8], [9], mainly concentrates on various issues of the target. The combined optimization and routing are not taken into account even though the main aim is energy reduction. In [12] the proposed algorithm corresponds to the compression of the data source in the initial section of the research work, further routing is carried out by employing a flow-based model. We implement a heterogeneous network where the input nodes possess various detection and transmission abilities, however, it is impossible to accumulate the data flow from various sources. The network of multimedia sensors presents a few examples of these types of the network [2]. In the current research work, we propose a combined optimization method for routing and encoding. The proposed algorithm relies on ADMM resulting in a completely allocated system.

A heterogeneous distributed-edge framework has been specifically formed by many public and private networks by offering support to the application of the Internet of Things. The network operators present a Network Function Virtualization (NFV) that distinguishes the operations of the network from specific hardware by operating the features in an adaptable software such as Virtual Network Functions (VNFs) that is operated on specific hardware [14]. The network providers are offered a unique chance that is associated with Software-Defined Networking (SDN), to set up the architecture to satisfy the requirements of a specified application [15]. Recently in fundamental cloud conditions, the research works mainly concentrates on the drawbacks of Network Function Virtualization. One of the disadvantages involved in cloud computing is end-to-end dormancy which is due to the impact of the physical gap between the cloud service providers and a heterogeneous distributed-edge framework that has been specifically formed by many public and private networks by offering support to the application of Internet of Things. The network operators present a Network Function Virtualization (NFV) that distinguishes the operations of the network from specific hardware by operating the features in an adaptable software such as Virtual Network Functions (VNFs) that is operated on specific hardware [16]. The network providers are offered a unique chance that is associated with Software-Defined Networking (SDN), to set up the architecture to satisfy the requirements of a specified application [17]. One of the disadvantages involved in cloud computing is end-to-end dormancy which is due to the impact of the physical gap between the cloud service providers and an efficient technique is developed for the ideal decision making policy and enhance the advantages for a long period. The level of accuracy and recurring is minimized by combining the efficiently handling feature of deep learning with reinforcement learning which is having decision-making capacity [19]. Deep learning is considered as one of the key technology in achieving self-adjusted SFC enhancement because of the benefits of self and virtual learning. Cloud computing that is generally associated with Software-Defined Networking and Network Function Virtualization are employed in various application of IoT to guarantee the efficiency of the service provider. A security framework which is based on cloud computing is introduced in [20] is employed to safeguard the Software-Defined vehicular applications of IoT. The blockchain nodes examine the real-time applications of the Internet of Things so that suspicious traffic and its behavior are updated by the blockchain and thus assuring data 
security and cost optimization. The attack of distributed denial of service (DDoS) has a significant effect on the exponential increase in the suspicious devices in the IoT applications. The model of decentralized secure DDoS collaboration solution (Cochain-SC) [21] was introduced by Z. Abou et al. to overcome the above issue by employing smart convention to the model. The main advantage of the Cochain-SC is that the blockchain enables privacy and cost-effective distributed collaboration among various Software-Defined Networking to reduce the attacks. P. K. Sharma et al. introduced a new Software-Defined-cloud architecture consisting of three layers [18] which are based on blockchain to overcome the drawback of security for outsourcing data and hence creating trust between consumer and the service providers. The request services and public set up is examined by the device layer. The distributed resources and enabling data operation are analyzed by a cloud layer. The computational resources are carried to the edge of the IoT network which is based on blockchain and Software-Defined Networking in the fog layer. The architecture of the distributed cloud which is based on the blockchain overcomes the drawback of privacy and decentralization. A unique structure combined with SDN that is used for the application of IoT is introduced by M. Pourvahab et al. in [21]. To assure secure synchronization between various SDN controllers for a network $\mathrm{H}$. Tang et al. in [20] addressed a consensus protocol that is based on the blockchain for Software-Defined Industrial Internet. Blockchains are widely used in many distributed applications because of their service trustworthy, cost-effective, and transparent nature [14].

\section{REALTED WORK}

The most widely considered technique in the research work of wireless mesh and sensor networks is Routing. The routing protocol that directs any routing path in the network of the wireless sensor to an individual or many defined base stations is termed as CTP [7]. Some of the applications of CTP are research, training, and an industrial process. The development of RPL [8] has been ensured by the perception of CTP. High efficiency is not achieved by the routing protocols such as CTP and RPL which is based on the tree because it is unable to generate the data set routes described in the wireless HART. Since packet loss is the main issue of these two protocols they are not applicable for the basic applications of industries. Therefore, to overcome the above problem, a multipath routing protocol is introduced (example, [11]-[15]) which improves the efficiency between the sender node and receiver node by enabling cluster-head disjoint or connection-disjoint. However, the energy utilization and load in the traffic are maintained between different network nodes by the introduction of routing protocol which is based on multipath RPL (example, [16]-[20]). When compared to other protocols, the data set routing mentioned in Wireless HART ensures high efficiency by considering the significant level of routing latency for the network of TSCH.

The application of a typical wireless HART has illustrated the reliability of employing its basic approaches for almost ten years and ensuring accurate low-power wireless communication in the processing site. A set of algorithms were proposed by Han et al. [21] and Wu et al. [22] to generate the data set path in a unified manner whereas, with the application of the Bellman-Ford algorithm, Modekurthy et al. introduced the generation of data set paths in a distributed manner [23]. In contrast to these techniques, a basic distributed routing protocol that is based on RPL is designed to produce and work with the data set paths. The overall solution for the network is obtained by designing a strategy of transmission scheduling which is operated just above the designed protocol. The analysis of transmission scheduling is considered as the prime concern for the applications of Wireless HART networks which are based on time [24]-[27]. The above-discussed scheduling methods are unified alternatives that are intended to operate on a data-centric network manager with data set routing operation. Also, researches have been made for the development of RPL networks with distributed scheduling [10], [27]-[32]. Consider an example of Orchestra in RPL networks where the nodes can determine their schedules which are introduced by Duquennoy et al. [10]. To ensure the proper utilization of IPv6 which facilitates load above the IEEE 802.15.4e TSCH networks is regulated by the operating group of $6 \mathrm{TiSCH}$ developed by IETF [9]. On the other hand, the experimental results of our analysis illustrated that the network operating with RPL experiences more recovery time and reduced efficiency when node malfunction and external interference take place. Synchronous transmissions [22]-[27] are considered as one more area of research. But for handling the synchronous transmission there is a necessity for a unified node in this transmission.

N. Michelusi et al. [28] presented an algorithm for Energyopportunistic Weighted Minimum Energy (E-WME) concerned with the routing techniques of "energy-harvestingaware" and thereby determining the individual sensor node overhead by employing the rate of energy harvesting and the energy available. A routing algorithm for Randomized Minimum Path Recovery Time (R-MPRT) is introduced in the research work of [28]. Therefore, the cost measured for a system can be expressed as the amount of energy consumed by the packet processing node (also known as energy packet) to the rate of energy harvesting. By considering the specified cost metric, the best optimal path to the destination is determined by the node. Further, the destination receives the data packet through a low-cost connection from the source node. An algorithm for R-MPRT is introduced by Hasenfratz et al. in [29] which is developed by making use of the energy remaining at the node rather than using the energy harvesting rate to attain an optimal solution. The decision of routing is considered depending on the cost measurements that consist of energy consumption and harvested energy ruined because of overloads which are explained in [13]. The algorithm for Distributed Energy Harvesting Aware Routing (DEHAR) is presented in [30] this algorithm determines the best optimal path to reach the destination by employing the energy available at every individual node and hop counts of the node. The perception of the Energy harvesting algorithm employs a local charge for every individual node that is constantly 
rationalized and is inversely related to the energy available at the node.

A Routing Protocol for Energy Harvesting Aware Ad-hoc OnDemand Distance Vector (AODV-EHA) is introduced by Gong et al. in [31]. The algorithm of AODV-EHA deals with wireless sensor networks by acquiring the benefits of the previous Ad-hoc On-Demand Distance Vector protocols. This algorithm calculates the best optimal path with low-cost transmission by making use of efficient energy harvesting techniques. The protocol required for smart energy harvesting routing (SEHR) is presented in [32]. The algorithm of SEHR initially considers the energy accessibility at the node, expected rate of energy, and approximate the energy collected from the renewable sources ( for example solar and radiofrequency) during the process of selecting the path. A routing protocol for energy-harvesting-aware that is based on the topology control scheme is introduced in [33]. Therefore, to speed up the topology of the network, the energyharvesting-aware protocol uses the method of game-theoretical to evaluate the status of energy and extracting capacity of every individual node. The power consumption of the node is collectively evaluated by itself or by the adjacent node energy resources and further examines the energy produced and extracted at every individual node with various time intervals. Although routing algorithms that are discussed above minimizes the energy consumed and increases the durability of nodes but still these algorithms possess some drawbacks. The feature of energy harvesting introduced in [10] and [12] will not consider the actual amount of energy extracted. The concept proposed in [12] and [18] employs perpetual replacement rates for all the nodes present within the network. Therefore, almost all existing algorithms are unable to interact with the probabilistic feature of the resources that renewable because of the inadequate extraction of energy. One more drawback is the implementation of a single energy source to obtain energy. It is indicated that renewable energy sources are supported by peak/off-peak intervals, climatic changes, seasons, and day and night rotations. Thus in real-time, the application of a single energy source may not be feasible to increase the longevity of the node. As a result, a novel routing algorithm for energy-harvesting aware [34] is developed by considering various types of energy extracting algorithms. In the case of the stratified environment, the overall data is incapable of adjusting to the differences in the energy status of the sensor nodes (for example the remaining, utilized, and collected energy levels). Thus, it is required to create tables for routing by making use of local data in a decentralized manner for the applications of the Internet of things [35]. The framework of efficient routing which is known as directed diffusion is presented by C. Intanagonwiwat. et. al [38] and is utilized for the networks of the sensor. This framework illustrates the concept of data-centric along with the application of data input transmission and encoding. The algorithm of hierarchical clustering mainly focuses on distributed activity, the requirements for communication that are asymmetric, and energy consumption in the networks of the sensor is addressed by Estrin et. al [36]. Jiang et. al in [39] introduced a routing protocol for the networks of mobile ad- hoc and is termed as a cluster-based routing protocol (CBRP). The nodes of a network are split into non-overlapping and interesting nodes that in a decentralized manner with a twohop diameter. On the other hand, the CBRP protocol is not appropriate for the sensor networks that are energy limited. LEACH is a hierarchical clustering algorithm presented by Heinzelman et. al [37].

\section{PROPOSED METHODOLOGY OF WSN FOR EFFECTIVE SCHEDULING AND ROUTING}

The technique of energy harvesting is considered as one of the viable strategies that are based on physical-layer security. This technique protects data from surveillance without necessitating upper-layer data encryption as well as significantly enhances the performance of wireless networks. On the other hand, this strategy introduces many difficulties because the opposing parties can listen to the confidential data that is being transmitted between the sender as well as the recipient through a relay. As a result, for energy harvesting, the signal's transmission power must be sufficiently high but to prevent eavesdropping, the transmission power must be low. Multi-hop multipath wireless networks make this much more complex. The shortest path selection protocol, best path selection protocol, as well as random path selection protocol, are the three protocols introduced in this research work. In the context of unauthorized parties as well as hardware failure, these protocols help in improving the security of multi-hop multipath randomize-and-forward cooperative wireless sensor networks where the source, as well as relay nodes, can harvest energy from the signal for transmitting data. Additionally, accurate closed-form expressions as well as the asymptotic outage probability for every protocol is been developed for various eavesdropping attacks. The theoretical outcomes are validated by the simulation results.

Particularly when considering a large number of nodes, the conventional battery charging or replacing for wireless devices is difficult as well as environmentally harmful. As a result, unique approaches that are based on renewable energy sources must be developed to reduce large power consumption. One of the simple, as well as ecologically beneficial techniques, is wireless energy harvesting which is used for extending the life of networks by harvesting energy from radio-frequency (RF), and by using specialized devices the signals are converted into power. The signal produced in the same or different networks could be used to extract this RF energy. On the other hand, solar-powered dedicated transmitters can be used if the harvested energy value is insufficient. Therefore, we can achieve a better result by combining zero-energy network operation as well as ease in wireless device charging. Furthermore, a thorough analysis must be carried out to ensure that good communication performance. The impacts of wireless energy harvesting from cooperative network transmissions on probability coverage as well as network durability are described. We examine the probability of connectivity using battery-less nodes that are driven by customized RF energy transmitters in the subsequent part of the research work. As shown in Fig. 2, by harvesting power from the RF transmissions of other wireless devices, or in 
some situations where power transmitters are placed especially for this function is feasible to extend the lifetime of lowpower, wireless devices via WEH. On the other hand, the WEH-enabled devices' communication efficiency must also be examined. The primary SWIPT-enabled WEH methods are explained in this section and are shown in Fig. 2.3. This technique can be classified as context-aware or contextunaware.

The performance of RF-to-DC conversion is a significant hardware constraint that affects the energy harvester. The RF energy that is converted into DC power is not lossless. To accommodate for these impairments, the efficiency of RF-toDC conversion as shown in Fig. 2.4, describes the energy receiver's potential which is highly reliant on the power received. Generally, the conversion efficiency improves linearly as well as in concave form for low input power values as well as for higher power values respectively. In prior research works, the performance of RF-to-DC conversion was assumed to be constant as well as was independent of the instantaneous amplitude irrespective of the received RF signal. Due to small changes in the received power, the above condition may work for randomly placed low-density networks i.e., for intensity 0.05 Devices $/ \mathrm{m} 2$, but it is not feasible for denser networks with $>0.05$ Devices $/ \mathrm{m} 2$. As a result, the performance of RF-to-DC conversion characteristics must be taken into account while developing upcoming WEH systems. In wireless, routing protocol has become a major role in terms of power consumption, packet delivery ratio and transmission of packet and its packet scheduling, but in the development in wireless communication, there are new protocols such as Collection Tree Protocol (CTP) for better improvement in latency and throughput and Routing Protocol for Low Loss Network and Power (RPL ${ }^{2} \mathrm{NP}$ ) which is based on IPv6 IEEE standard discussed in [7-9]. These two advanced protocols is replacement of routing centralization and scheduling algorithms in WSAN's in industry. These protocols are combined together by considering the the advantages of both and it is named as Autonomous Scheduling and Distributed Graph Routing (DDSR) which will take care of automatic routing and transmission of packet between source and destination in a disturbed mode. The following are the main contribution of this research work

1. Development of low power and low lossy networks routing protocol with help of RPL ${ }^{2} \mathrm{NP}$ which will operates on graph routing and scheduling through minimum latency and high throughput.

2. Design of two scheduling approaches to compute automatic transmission based on the routing graph. The first approach is to find minimised distance between source and destination for end-to-end latency and eliminates the conflicts between other packet and it is to minimize the traffic in real world scenario.

3. The proposed $\mathrm{RPL}^{2} \mathrm{NP}$ is an oriented distance based routing protocol for development of low power IPv6 network. The working principles of $\mathrm{RPL}^{2} \mathrm{NP}$ is as follows.

The scheduling of packet and transmission is totally based on Internet Access Point (IAP) and each node has rank and its rank allocation is purely based on distance to the destination using cost function i.e excepted transmission count (ETC) and then packet is forwarded towards route to neighbor node. The generation of routes by RPL ${ }^{2} \mathrm{NP}$ are not in graph route initially because every node has only one preferred "head" in the whole network to use many head's, suppose those head's equally preferred and have same or identical rank in the network then there are choice to get collapse or interference occurring within the network and it leads to lose of packet. To overcome this issue, the modified RPL ${ }^{2} \mathrm{NP}$ routing network assigns two preferred head's to each node at a time as default routes and forms the routing graph in the network as per following specifications.

Directed Routing Graph (DRG): it forms the routing among all nodes without co-exists which are wanted communicate with other for packet transmission. This routing i.e all selected links for routing orient toward the neighbor or terminates at the destination or access points for ensuring that data or messages should delivered safely to the destination without any co-exist in the graph. The proposed Directed Routing Graph is more efficient routing algorithm compared with AODV, DSR and OLSR protocols for minimization of delay and congestions and based results obtained the DRG protocol has more throughput and high efficient as shown Table.1.

Best head and second best head selection: This selection alterative solution for avoiding of co-exists or interfering of packet, in the network has best head and second head, the best head is to locates on the main path from node to access point with shortest distance from destination node and second head has another best shortest distance path from the same destination node to serve as backup routing so that packet delivery ratio can be increased.

Allocation of Rank: Every node has a rank and all access points allocates their own rank, initially it is ' 1 ' and based on the best head's, the initially assigned rank is updated by increasing by ' 1 '.

Weighted ETC: The cost function of weighted $\left(\mathrm{ETC}_{\mathrm{w}}\right)$ is the node to measure the distance from the access point by using two routings based on equation (1)

$$
\mathrm{ETC}_{\mathrm{w}}=\mathrm{W}_{1} * \mathrm{ETC}_{\mathrm{acc}}+\mathrm{W}_{2} * \mathrm{ETC}_{\mathrm{accs}}
$$

Where $\mathrm{ETC}_{\mathrm{acc}}$ is the distance accounted ETC from access point with help of best head. ETC $_{\text {accs }}$ is the distance accounted ETC from access point with help of second head. $\mathrm{W}_{1}$ and $\mathrm{W}_{2}$ are two best heads weighted and these are given in equations (2) and (3).

$$
\begin{aligned}
& W_{1}=1-\left(1-\frac{1}{E T C_{b h}}\right)^{2} \\
& W_{2}=1-\left(\frac{1}{E T C_{b h}}\right)^{2}
\end{aligned}
$$

Where $E T C_{b h}$ represents ETC between the source node and its best head. As per standard wireless communication discussed in [5], the transmission of first packet through best head and the retransmission of second packet through second best head which is backup route. So $\mathrm{W}_{1}$ is probability of successfully delivered packet at time of first two transmission attempts, $\mathrm{W}_{2}$ is probability of unsuccessful attempts fail. All the nodes present in the network are broadcast their own ranks periodically to join into transmission mode and based on it, the ranks are allocated. After allocation of ranks as best head and second head, "Joined callback message" sends to the selected 
best head and second best head and also it informs bout the selection to all other nodes.

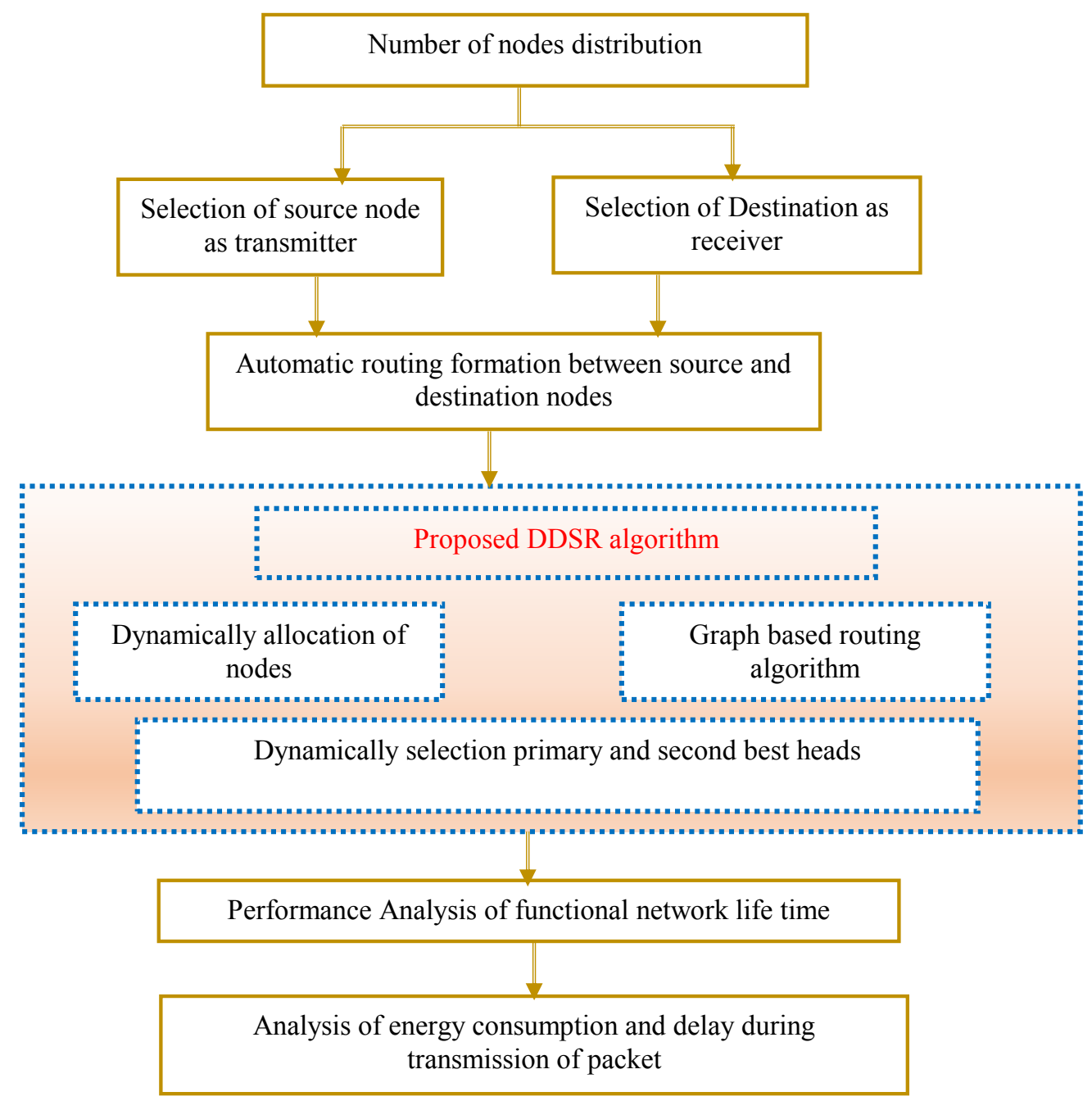

Fig.1. Working Flow diagram of Proposed Dynamically Routing using DDSR for low energy consumption and high throughput
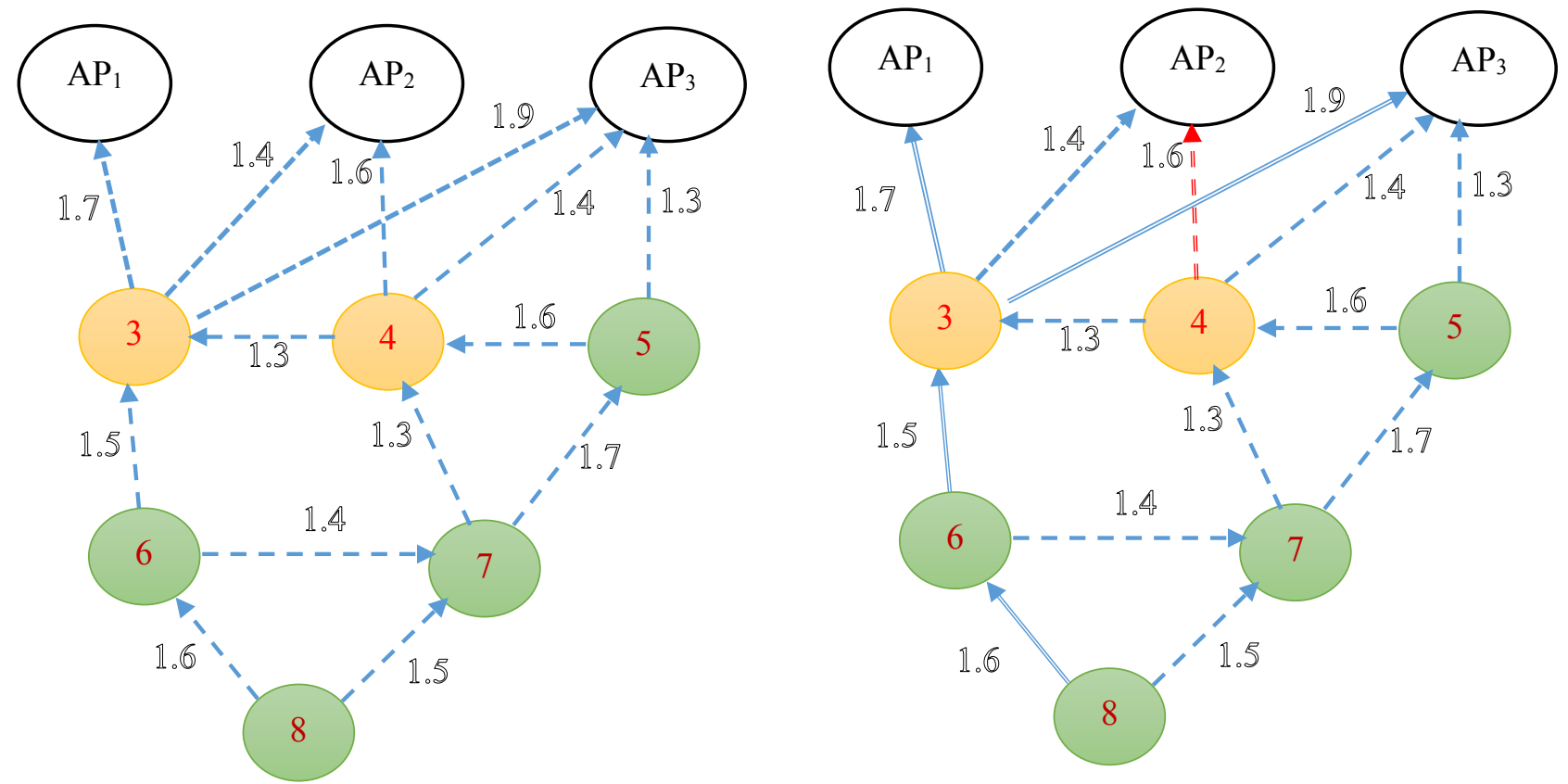
Fig.2. Proposed created network topology for three access points and six field nodes and their transmission paths and directions. (a) Created network topology (b) Routing Graph and best and second head's.

Algorithm 1: Dynamically Distributed \& Schedule Routing (DDSR)

$$
\begin{aligned}
& \text { Input }: \mathrm{R}_{\mathrm{id}}, \mathrm{N}_{\mathrm{id}} \\
& \text { Output }: \text { Updated router table }\left(\mathrm{R}_{\text {table }}\right) \\
& \text { Initialization }: \mathrm{R}_{\text {table }}=\mathrm{NULL}, \\
& \operatorname{ETC}_{W}\left(\mathrm{~N}_{\text {id }}\right)=\operatorname{Rank}\left(N_{\text {id }}\right)=\infty
\end{aligned}
$$

Condition 1: if $\mathrm{N}_{\mathrm{id}}=\mathrm{R}_{\mathrm{id}}$ then

////Initialize the access point

Compute Rank=1 and $\mathrm{ETC}_{\mathrm{w}}=0$;

Broadcast about join-in messages;

end

Condition 2:if $\operatorname{Rank}\left(\mathrm{N}_{\mathrm{id}}\right)=\infty \& \sim \mathrm{N}_{\mathrm{id}}=\mathrm{R}_{\mathrm{id}}$ then

/////Allow receiver to receive the first join-in message from i

Compute $\mathrm{ETC}_{\mathrm{acc}}\left(\mathrm{N}_{\mathrm{id}}, \mathrm{i}\right)=\mathrm{ETC}\left(\mathrm{N}_{\mathrm{id}, \mathrm{i}}\right)+\mathrm{ETC}_{\mathrm{w}}(\mathrm{i})$;

Compute sender message as its bes head;

Compute $\mathrm{ETC}_{\min }=\mathrm{ETC}_{\mathrm{acc}}\left(\mathrm{N}_{\mathrm{id}}, \mathrm{i}\right)$;

Compute Rank $\left(\mathrm{N}_{\mathrm{id}}\right)=\operatorname{Rank}(\mathrm{i})+1$;

Transmit joined message callback; end

Condition 3: if $\operatorname{Rank}\left(\sim \mathrm{N}_{\mathrm{id}}\right)=\infty \& \sim \mathrm{N}_{\mathrm{id}}=\mathrm{R}_{\mathrm{id}}$ then

$/ / / / /$ Receiver, receivers the non first messafe join-in from I

Compute $\mathrm{ETC}_{\mathrm{acc}}\left(\mathrm{N}_{\mathrm{id}}, \mathrm{i}\right)=\operatorname{ETC}\left(\mathrm{N}_{\mathrm{id}}, \mathrm{i}\right)+\mathrm{ETC}_{\mathrm{w}}(\mathrm{i})$;

If $\mathrm{ETC}_{\mathrm{acc}}\left(\mathrm{N}_{\mathrm{id}}, \mathrm{i}\right)<\mathrm{ETC}_{\min }$ then

Compute it as best head as the second best head;

Compute sender message as best head;

Compute $\mathrm{ETC}_{\min }=\mathrm{ETC}_{\mathrm{acc}}\left(\mathrm{N}_{\mathrm{id}, \mathrm{i}}\right)$;

Compute Rank $\left(\mathrm{N}_{\mathrm{id}}\right)-\operatorname{Rank}(\mathrm{i})+1$;

Transmit as joined callback message;

end

Condition 4: if $\mathrm{ETC}_{\mathrm{acc}}\left(\mathrm{N}_{\mathrm{id}}, \mathrm{S}_{\mathrm{bh}}\right)>\mathrm{ETC}_{\mathrm{acc}}\left(\mathrm{N}_{\mathrm{id}}, \mathrm{i}\right)>=\mathrm{ETC}_{\min }$ and $\operatorname{Rank}(\mathrm{i})<\operatorname{Rank}\left(\mathrm{N}_{\mathrm{id}}\right)$ then

Compute sender, sends message as second best head;

Transmit joined message as joinback; end

$\mathrm{ETC}_{\mathrm{w}}\left(\mathrm{N}_{\mathrm{id}}\right)=\mathrm{W}_{1} * \mathrm{ETC}_{\mathrm{acc}}\left(\mathrm{N}_{\mathrm{id}}, \mathrm{S}_{\mathrm{bh}}\right)+\mathrm{W}_{2} * \mathrm{ETC}_{\mathrm{acc}}\left(\mathrm{N}_{\mathrm{id}}, \mathrm{S}_{\mathrm{bh}}\right) ;$

Broadcast message as join-in; end

Condition 5:if Receive joined message callback then

Update the router table $\mathrm{R}_{\text {table }}$ and sender message is added as sub router;

end

The proposed distributed graph shown in algorithm 1 and it start with access point to form the routing graph and it routes towards the access pint. Before network starts, the graph initialize the rank to 1 and $\mathrm{ETC}_{\mathrm{w}}$ to 0 , therefore, the network starts broadcasting join-in message. The remaining nodes computes their rank and $\mathrm{ETC}_{\mathrm{w}}$ to infinity. Suppose any node receives the join-in messages from any another nodes, it opts its best head and second head purely based on accounted ETC routing table values and then it compute its rank by raising its best heads rank by 1 . After updating of heads ranks, the node starts broadcasting join-in message to another nodes. The ETC initialization between any two nodes are decided purely based on Strength of Received Signals (SRS). In this work, we have set $\mathrm{SRS}_{\min }=-75 \mathrm{dBm}$ and $\mathrm{SRS}_{\max }=-90 \mathrm{dBm}$, when $\mathrm{SRS}$ value is more than $-90 \mathrm{dBm}$, then ETC is set to 1 otherwise ETC is set to 5 , and the ETC can scaled randomly netween 1 and 5 . In case, there is transmission error occurs between range 1 and 5 , it can be measured by using equation (4).

$$
\text { ETC }=\text { ETC }_{\text {old }} * \beta+q *(1-x)
$$

Where $E T C_{\text {old }}$ is the ETC value applied before maximum error occurs, $\mathrm{q}$ is error coeeficients and $\beta$ is weight factor between 0 and 1. The Fig.2. shows the data paths examples for packet transmissions that has three Access Point (AP) and six field nodes. The dash lins shows the ETC values with links. Whenever network starts the packet transmission, the three $\mathrm{AP}_{1}, \mathrm{AP}_{2}$ and $\mathrm{AP}_{3}$ are starts broadcasting their $\mathrm{ETC}_{\mathrm{w}}$ values and ranks to neighbour nodes. \#3 selcts $\mathrm{AP}_{3}$ as its best head and $\mathrm{AP}_{1}$ as its second best head, the selection of head's are based on $\mathrm{ETC}_{\mathrm{acc}}$ values because $\mathrm{ETC}_{\mathrm{acc}}\left(3, \mathrm{AP}_{3}\right)$ is greater than $\mathrm{ETC}_{\mathrm{acc}}\left(3, \mathrm{AP}_{1}\right)$. Similary $\# 4$ selects $\mathrm{AP}_{2}$ as its best head and $\mathrm{AP}_{3}$ as second best head since $\mathrm{ETC}_{\mathrm{acc}}\left(4, \mathrm{AP}_{2}\right)$ is greater than $\mathrm{ETC}_{\mathrm{acc}}\left(4, \mathrm{AP}_{3}\right)$. Therefore the rank of both \#3 and \#4 are 2 and these are starts broadcasting their rasnks as join-in measssage to neighbours as shown in Fig.3. To avoid loops, the \#3 and \#4 are not selected th link between \#4 and \#5. Based on connectivity among selected heads and neighbour nodes, the routing graph is generated and it is shown in Fig.1. The solid lines are denoted the major path (primary) i.e $\# 8 \rightarrow \# 6 \rightarrow \# 3 \rightarrow \# A P_{1}$ and the dash lines denotes the backup routes

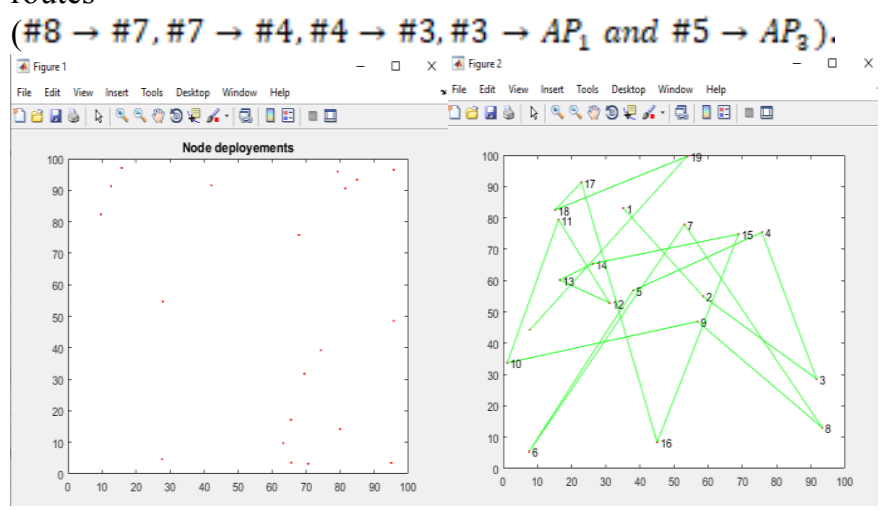

(a)

(b)

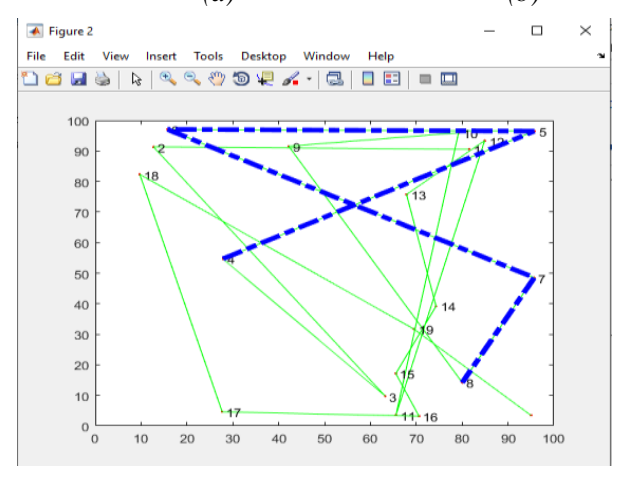


(c)

Fig.3. Proposed Wireless Sensor Network, nodes deployment and path establishment between source node and destination node. (a) 20 Nodes deployment (b) Connection establishment among all nodes (c) Path establishment between source and destination nodes.

Slots allocation for application as shown in Fig.1.: There is choice of attempting multiple transmission through scheduling for each and every packet with help of major path and backup path. Therefore, the transmission and reception of packet or schedule are purely depending on their unique id which is assigned to each and every node. All these id's are generated as integer byte and stored in LUT and mapped as MAC address. The allocation of slots (s) is given in equation (5).

$s=N *\left(N_{o d e} e_{i d}-M_{A P}\right)-N+\beta$

Where $\mathrm{N}$ is no of attempts for transmission for each packet, $M_{A P}$ is no of access points, Node $e_{i d}$ is neighbour node id from routing table and $\beta$ is $s^{\text {th }}$ slot in the application for $\beta^{\text {th }}$ transmission attempts. To increase throughput of the DDSR in WSN, the hybrid WSN which includes Dijkstra algorithm, minimum tree spanning and localized minimum spanning are incorporated for finding of shortest path between source and destination. In these hybrid algorithms mainly depending on message and node id's which are based on locally best decision of each and every node and it has its own information and this will be shared with neighborhood to find the shortest path in the graph in terms of best and second head's. By reducing of distance among the source and destination nodes, the throughput drastically increased and minimized the number dead nodes as shown Fig.5. With help of Dijkastra algorithm, the problem of maximum distance between source and destination are minimized and step by step process is shown in algorithm 2.

Algorithm 2: Shortest path identification between source and destination nodes

Step 1: Parameters initialization

Iteration number $=0$, distance $=$ any number in positive infinity and data set $[i]=0$, where $i=0,1,2,3 \ldots \ldots . . n-m)$.

Notations: $\mathrm{r}_{\mathrm{n}}=$ radius of transmission

$\mathrm{sv}=$ starting of the node

$\mathrm{n}_{\mathrm{r}}=$ node relay

$\mathrm{r}_{\mathrm{n}}=$ Receiver node

Step 2: When $\cos (\mathrm{e})[\mathrm{sv}] \quad[\mathrm{i}[<\mathrm{r}$ then data $\operatorname{set}[\mathrm{i}]=1,(\mathrm{i}=0,1,2,3 \ldots \ldots \mathrm{m})$

Compute $\cos [\mathrm{i}][\mathrm{j}]$ for distance between source node (i) and destination node $(\mathrm{j})$

Step 3: If data $\operatorname{set}\left[\mathrm{r}_{\mathrm{n}}\right]=1$ then

Compue distance $=\cos (e)[\mathrm{sv}]\left[\mathbf{r}_{\mathbf{n}}\right]$

else

go to step 4

Stept 4: All values of $\mathrm{i}$ are belongs to $\{$ data set $[\mathrm{i}]=1\}, \mathrm{n}_{\mathrm{r}}$ $[i]=1$, data set $[j]=1$ when $\mathrm{j}$ also belongs to $\left\{\cos (e)\left[\mathrm{n}_{\mathrm{r}}[\mathrm{j}]<\mathrm{k}\right\}\right.$ for all values og $\mathrm{j}=0,1,2,3 \ldots \ldots \ldots \ldots$ )

else

record the distance that falls in data $\operatorname{set}\left[\mathbf{r}_{\mathbf{n}}\right]=\mathbf{1}$ and compue the distance between nodes is $\cos (\mathrm{e})[\mathrm{sv}]$ $\mathrm{n}_{\mathrm{r}}[0]<\sum_{j=0}^{i-1} \cos (e)[s v][\operatorname{mr}[(\mathrm{j}+1) \cos (\mathrm{e})[\operatorname{mr}(\mathrm{i}(\mathrm{sv}))]$ end

\subsection{Measurement of Energy in WSN}

After establishment of paths among the heads and other field nodes, the energy is measured per packet transmission as per following specifications and ploted the obtained energy for without DDSR and with DDSR.

Sinks: sink. $\mathrm{x}=1.5 *$ WIDTH and sink. $\mathrm{y}=0.5 *$ HEIGHT

Number of Nodes in base station area is $n=20$

Probability of a node is $p=0.2$

Battery capacity is $\quad \mathrm{Eo}=0.1$,

$\mathrm{ETX}=50 * 0.000000001, \mathrm{ERX}=50 * 0.000000001$

Transmission energy $\mathrm{Efs}=10 * 0.000000000001$ and

Emp $=0.0013 * 0.000000000001$.

Data Propagation Energy is EDA $=5 * 0.000000001$

Thresholod for transmiting data to SINK are $\mathrm{h}=100$ and $\mathrm{s}=2$

\section{RESULTS AND DISCUSSION}

The Fig.5. Shows the performance analysis between different paramedics and their optimization shown in Fig.4. The DDSR able to minimized the end to end delay during the transmission of packets from source node to destination node, Fig.5.(a) shows the optimization of delay between proposed DDSR algorithm and existing algorithm, the red color shows the end to end delay for existing algorithm and yellow color shows the proposed algorithm delay and it is concluded that the delay of proposed communication for packet transmission is optimized $14 \%$ compared to existing work. The Fig.6 (b) shows the number of packets lost during the packets transmission and due to effective scheduling and formation routing table, the losses are able to minimize and compared to existing results shown in [12].

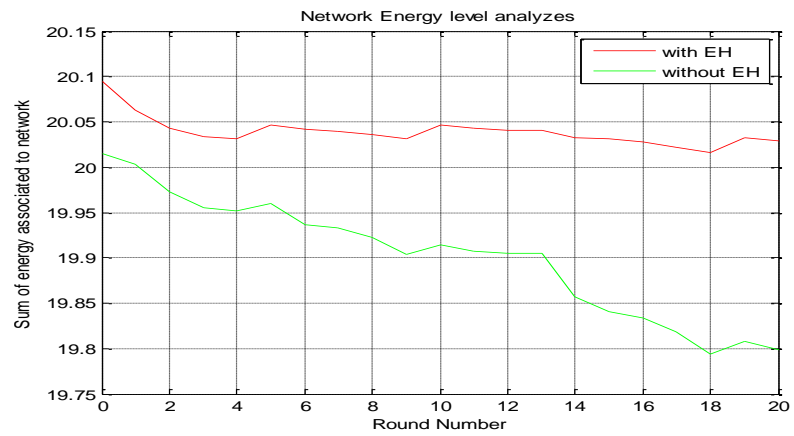

Fig.4. Energy consumption per round per packet transmission between with DDSR-EH and without DDSR-EH.
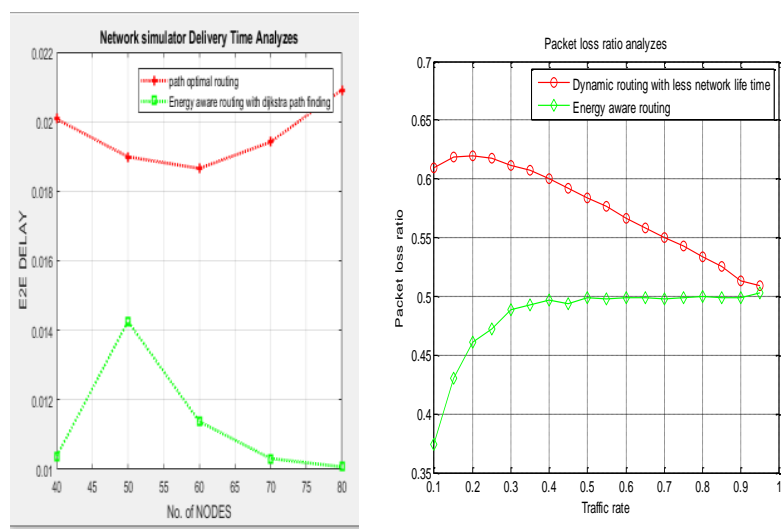
(a)

(b)

Fig.5. Performance analysis of End-to-End delay, packet loss ration with and without of dynamic routing (a) End-to-End delay (b) Without dynamic routing and with dynamic routing.

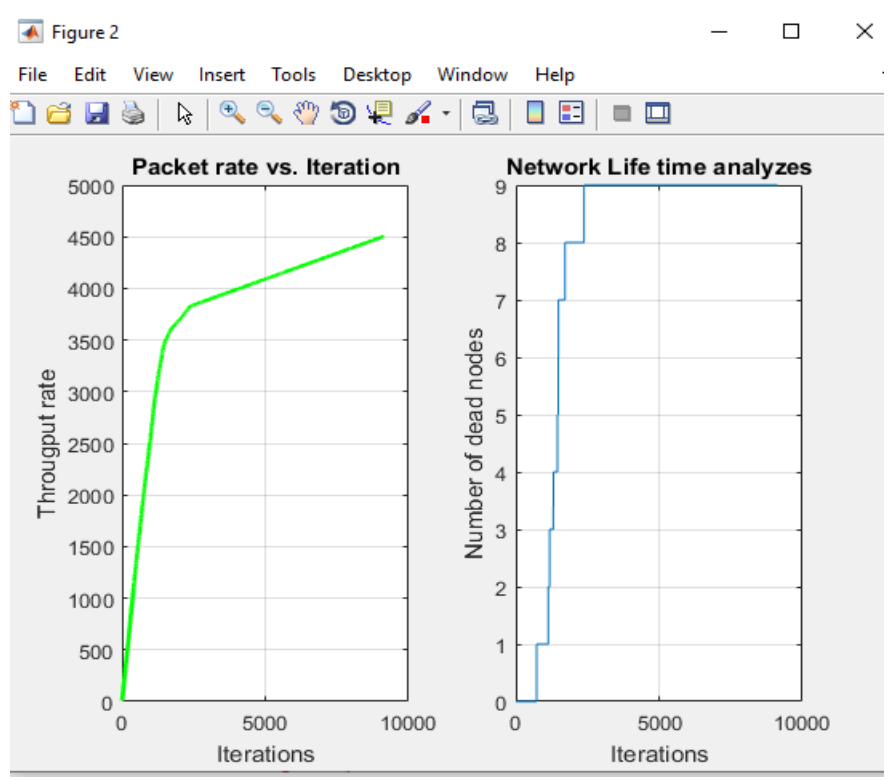

Fig.6. Performance analysis of throughput and number of dead nodes w.r.t life time of network of DDSR during transmission of packets.

In proposed system, DDSR as feasible design flow to model the energy hravesting thorugh both solar and RF fading channel. An energy received and storage by the receiver antenna under different noisy channel like Rayleigh fading enviornment is simulated in MATLAB 2017a using communication system toolbox. The modeled channel is accessed with help of command i.e energy_channel= rayleighchan $\left(T_{s}, D_{m}\right)$ where $T_{s}$ is sampling frequency of $1000 \mathrm{~Hz}$ and $\mathrm{D}_{\mathrm{m}}$ is doppler maximum shift of $25 \mathrm{~Hz}$ and its correspondings to rayleigh fading channel as shown in Fig.7. The received signal power harvested by a RF rectifer with minimum loss efficiency and its energy harvesting at time $t$ is given by

$H_{e}(t)=\int_{0}^{1} P_{F}(\tau) d \tau$

Where $P_{F}$ received power at given timer. The $\left\{H_{\theta}(t), \mathrm{t}>0\right\}$ is the energy harvested during process of observing RF signals.

The Rayleigh RF channel $\left\{H_{e}(t), t>0\right\}$ has been modeled help of homogeneous to fulfill the following basic properties:

1. $P\left(H_{\theta}(0)=0\right)=1$

2. Increasing the $H_{e}(A+s)-H_{e}(A)$

3. An energy harvested in a given interval of magnitude (A), $H_{\theta}(A+s)-H_{e}(A)$ is the distribution process with parameter $\alpha \mathrm{A}$ and $\beta$ along with density.

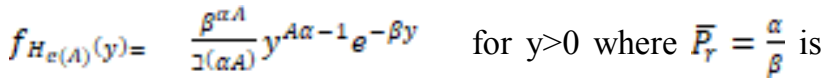
the mean received signal power

The harvested energy of mean and its variance in an interval of magnitude $\mathrm{A}$ is given by $\in\left[H_{e}(A)\right]=\overline{P_{r}} A$ and $\operatorname{var}\left(H_{e}(A)\right)=\alpha^{-1} A \overline{P_{r}^{2}}$
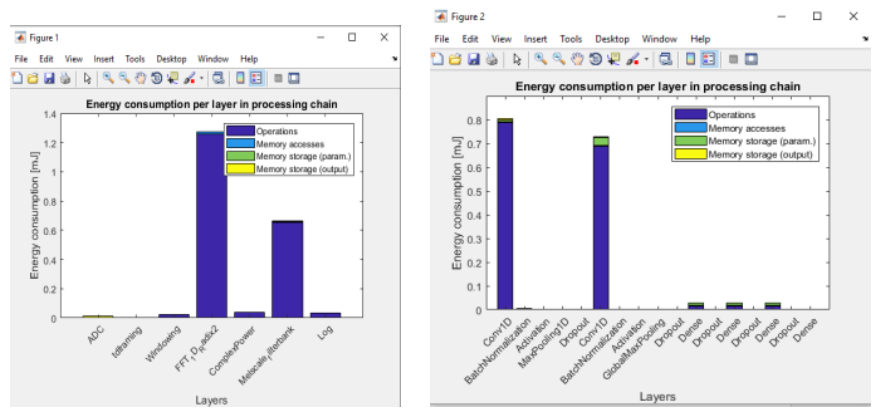

Fig.7 Energy saved through RF and Solar and consumed during the process DDSR.

The first thing that must be considered when harvesting energy is the source of the energy particularly where the energy source is located and the amount of energy available. When considering radio frequency (RF) energy there are many different options including radio, digital TV, cell phones, and Wi-Fi. To choose from. The power density and availability of the RF energy was considered in the tables below. Table 1 shows the power density in a given range for a $50 \mathrm{Kw}$ AM radio station which usually operates between $420 \mathrm{KHz}-1350$ KHz. Table 2 covers the power density and range for $95 \mathrm{~W}$ stations which operate at $628 \mathrm{MHz}$ to $862 \mathrm{MHz}$ and 1520 $\mathrm{MHz}$ to $2110 \mathrm{MHz}$.

Table 1. Power density available for a $50 \mathrm{~kW} \mathrm{AM} \mathrm{radio}$ station.

\begin{tabular}{|c|c|}
\hline \multicolumn{2}{|c|}{$50 \mathrm{~kW}$ AM Radio Station } \\
\hline Distance & Power Density Available \\
\hline $5 \mathrm{Km}$ & $159 \mu \mathrm{W} / \mathrm{m}^{2}$ \\
\hline $10 \mathrm{Km}$ & $40 \mu \mathrm{W} / \mathrm{m}^{2}$ \\
\hline
\end{tabular}

Table 2. Power density available for a 100 W GSM base station.

\begin{tabular}{|c|c|}
\hline \multicolumn{2}{|c|}{ 95W Base Station } \\
\hline Distance & Power Density available \\
\hline $100 \mathrm{~m}$ & $800 \mu \mathrm{W} / \mathrm{m}^{2}$ \\
\hline $500 \mathrm{~m}$ & $32 \mu \mathrm{W} / \mathrm{m}^{2}$ \\
\hline $1000 \mathrm{~m}$ & $8 \mu \mathrm{W} / \mathrm{m}^{2}$ \\
\hline
\end{tabular}




\section{CONCLUSION}

The contribution of this paper is on improvising of current WSAN and WSN networks for wireless communications and increasing od their scalability through effective routing and scheduling to enhance visibility and predictability of wireless network operation in WSN. This paper decentralizes the organization the board in Wireless DDSR and presents the primary circulated diagram steering and self-governing booking arrangement that permits the field gadgets to process their own chart courses and transmission plans. The Figures show the synopsis of contrasts among existing directing and booking calculations like DiGS/DiGS-CD contrasted with proposed Wireless DDSR. Test results from two physical testbeds and a huge scope recreation show our answer gives a huge enhancement for network dependability, dormancy, energy proficiency, and disappointment resilience under elements, basic properties for modern applications, over cutting edge at the expense of somewhat higher force utilization and longer organization instatement. In this paper, we have likewise explored the issues of energy proficiency and QoS in a consolidated way for heterogeneous WSN networks within the sight of three energy the executive's methods: to address the issues of varieties of traffic burden and energy accessibility conditions. We have then built up an "energy ease off" instrument, to be coordinated into WSN sensors for DDSR. The DDSR calculation can be executed in any IEEE 02.15.4 standard-based WSN applications with the least alterations. Reproduction results have exhibited that our proposed calculation essentially improves energy effectiveness while fulfilling the QoS prerequisites. The outcomes likewise show that the organization of the crossbreed setup with different fuel sources is an effective, compelling and pragmatic answer for at the same time improves the energy-proficient and QoS issues just as to expand the lifetime of gadgets in heterogeneous WSN networks. We examined the accessibility of a WPSN over various routing algorithms such as unicast, broadcast as well as fading scenarios in this research work. The computation of connectivity probability for every event is carried out mathematically by taking into account the probability of nodes that are active and further evaluated using Monte Carlo simulations. In addition, we examined various routing algorithms by considering battery-powered as well as battery-less nodes that harvest RF energy from PBs, also considering the conditions in which a WPSN is associated. We intend to continue this study in three different ways in future research work: i) using the variable performance of RF-to-DC conversion in the framework that offers more precise as well as genuine outcomes; ii) obtaining the best alternatives analytically by offering the highest connectivity, and the last way is to analyze PBs energy consumption and thereby determining the optimal parameters for an energy-efficient WPSN.

\section{REFERENCES}

[1] Arati Manjeshwar,et.al, "APTEEN: A Hybrid Protocol for Efficient Routing and Comprehensive Information
Retrieval in Wireless Sensor Networks", 1530-2075/02, IEEE, 2002.

[2] W. Heinzelman, A. Chandrakasan, and H. Balakrishnan, "uAMPS ns Code Extensions". http://wwwmtl.mit.edu/research/icsystems/uamps/ leach.

[3] W. B. Heinzelman. "Application-Specific Protocol Architectures for Wireless Networks". PhD thesis, Massachusetts Institute of Technology, June 2000.

[4] A. Manjeshwar and D. P. Agrawal. TEEN: A Routing Protocol for Enhanced Efficiency in Wireless Sensor Networks.In 1st International Workshop on Parallel and Distributed Computing Issues in Wireless Networks and Mobile Computing, April 2001.

[5] Michele Rossi,et.al, "Distributed Learning Algorithms for Optimal Data Routing in IoT Networks", IEEE Transactions on Signal And Information Processing Over Networks, 2373-776X, 10.1109/TSIPN.2020.2975369, VOL. 6, 2020.

[6] N. A. Pantazis, A. Spiridonos, S. A. Nikolidakis, and D. D. Vergados, "Energy-proficient directing conventions in remote sensor networks:Asurvey," IEEE Commun. Surv. Tut., vol. 15, no. 2, pp. 551-591, May 2013.

[7] D. Zordan, B. Martinez, I. Villajosana, and M. Rossi, "On the exhibition of lossy pressure plans for energy compelled sensor organizing," ACM Trans. Sensor Netw., vol. 11, no. 1, pp. 1-34, Nov. 2014.

[8] N. Cao, E. Masazade, and P. K. Varshney, "A multiobjective improvement based sensor choice strategy for target following in remote sensor organizations," in Proc. sixteenth Int. Conf. Inf. Combination, 2013, pp. 974-980.

[9] M. Centenaro, M. Rossi, and M. Zorzi, "Joint improvement of lossy pressure and transport in remote sensor organizations," in Proc. IEEE Globecom Workshops, Washington, DC, USA, Dec. 2016, pp. 1-6.

[10]P. Giselsson and S. Boyd, "Direct intermingling and metric determination for Douglas-Rachford parting and ADMM," IEEE Trans. Autom. Control, vol. 62, no. 2, pp. 532-544, Feb. 2017.

[11]A. Biason, C. Pielli, A. Zanella, and M. Zorzi, "Access control for IoT hubs with energy and constancy requirements," IEEE Trans. Remote Commun., vol. 17, no. 5, pp. 3242-3257, May 2018.

[12]F. Iutzeler, P. Bianchi, P. Ciblat, and W. Hachem, "Unequivocal union pace of a conveyed rotating course technique for multipliers," IEEE Trans. Autom. Control, vol. 61, no. 4, pp. 892-904, Apr. 2016.

[13] S. Javaid, H. Fahim, Z. Hamid, and F. B. Hussain, "Traffic-mindful blockage control (TACC) forwirelessmultimedia sensor organizations," Multimedia Tools Appl., vol. 77, no. 4, pp. 4433-4452, Feb. 2018.

[14] Shaoyong Guo,at.al, "Confided in Cloud-Edge Network Resource Management: DRL-driven Service Function Chain Orchestration for IoT", IEEE Internet of Things Journal, DOI 10.1109/JIOT.2019.2951593, 2327-4662 (c) 2019 IEEE.

[15]H. Hawilo, M. Jammal, and A. Shami, "Organization work virtualizationaware orchestrator for administration 
work affixing position in the cloud," IEEE Journal on Selected Areas in Communications, vol. 37, no. 3, pp. 643-655, March 2019.

[16] M. M. Tajiki, S. Salsano, L. Chiaraviglio, M. Shojafar, and B. Akbari, "Joint energy productive and qos-mindful way allotment and vnf position for administration work tying," IEEE Transactions on Network and Service Management, vol. 16, no. 1, pp. 374-388, March 2019.

[17] Junyang Shi.et.al, "Distributed Graph Routing and Scheduling for Industrial Wireless sensor-actuator Networks", 1063-6692, 2019 IEEE, IEEE/ACM TRANSACTIONS ON NETWORKING, VOL. 27, NO. 4, AUGUST 2019.

[18] L. Gu, D. Zeng, S. Tao, S. Guo, H. Jin, A. Y. Zomaya, and W. Zhuang, "Decency mindful powerful rate control and stream planning for network utility boost in network administration chain," IEEE Journal on Selected Areas in Communications, vol. 37, no. 5, pp. 1059-1071, May 2019.

[19] X. Chen, Z. Zhu, J. Guo, S. Kang, R. Proietti, A. Castro, and S. J. B. Yoo, "Utilizing blended system gaming to acknowledge motivation driven vnf administration chain provisioning in representative based flexible optical interdatacenter networks," IEEE/OSA Journal of Optical Communications and Networking, vol. 10, no. 2, pp. A232-A240, Feb 2018.

[20]C. Mouradian, S. Kianpisheh, M. Abu-Lebdeh, F. Ebrahimnezhad, N. T. Jahromi, and R. H. Glitho, "Application segment arrangement in nfvbased cross breed cloud/mist frameworks with portable haze hubs," IEEE Journal on Selected Areas in Communications, vol. 37, no. 5, pp. 1130-1143, May 2019.

[21]Z. Chen, S. Zhang, C. Wang, Z. Qian, M. Xiao, J. Wu, and I. Jawhar, "A tale calculation for $\mathrm{nfv}$ chain arrangement in edge figuring conditions," in 2018 IEEE Global Communications Conference (GLOBECOM), Dec 2018, pp. 1-6.

[22] Thien Duc Nguyen.et.al, “A Distributed EnergyHarvesting-Aware Routing Algorithm for Heterogeneous IoT Networks", DOI 10.1109/TGCN.2018.2839593, IEEE, IEEE Transactions on GREEN Communications and Networking, 2473-2400, 2018 IEEE

[23] P. K. Sharma, M. Chen, and J. H. Park, "A product characterized haze hub based disseminated blockchain cloud engineering for iot," IEEE Access, vol. 6, pp. 115124, 2018.

[24]F. Ferrari, M. Zimmerling, L. Mottola, and L. Thiele, "Low-power remote transport," in Proc. SenSys, 2012, pp. $1-14$.

[25]F. Ferrari, M. Zimmerling, L. Thiele, and O. Saukh, "Productive organization flooding and time synchronization with polished," in Proc. SenSys, Apr. 2011, pp. 73-84.

[26] O. Landsiedel, F. Ferrari, and M. Zimmerling, "Mayhem: Versatile and productive all-to-all information sharing and in-network preparing at scale," in Proc. SenSys, 2013, p. 1.
[27] M. Doddavenkatappa, M. C. Chan, and B. Leong, "Sprinkle: Fast information dispersal with useful impedance in remote sensor organizations," in Proc. NSDI, 2013, pp. 269-282

[28] M. Doddavenkatappa and M. C. Chan, "P3: A functional parcel pipeline utilizing simultaneous transmissions for remote sensor organizations," in Proc. IPSN, 2014, pp. 203-214.

[29] N. Michelusi and M. Zorzi, "Optimal adaptive random multiaccess in energy harvesting wireless sensor networks," IEEE Trans. Commun., vol. 63, no. 4, pp. 1355-1372, Apr 2015.

[30] T. D. Nguyen, J. Y. Khan, and D. T. Ngo, "An adaptive MAC protocol for RF energy harvesting wireless sensor networks," in Proc. of GLOBECOM'16, 2016.

[31]D. Hasenfratz, A. Meier, C. Moser, J. J. Chen, and L. Thiele, "Examination, correlation, and enhancement of directing conventions for energy reaping remote sensor organizations," in Proc. of SUTC'10, Jun 2010, pp. 1926.

[32] G. Martinez, S. Li, and C. Zhou, "Wastage-mindful steering in energy harvesting Remote Sensor Networks," IEEE Sensors J., vol. 14, no. 9, pp. 2967-2974, Sep 2014.

[33]P. Gong, Q. Xu, and T. M. Chen, "Energy collecting mindful directing convention for remote sensor organizations," in Proc. of CSNDSP'14, Jul 2014, pp. 171-176.

[34] J. Bai, M. Fan, J. Yang, Y. Sun, and C. Phillips, "Keen energy collecting steering convention for WSN based Ewellbeing frameworks," in Proc. of the 2015 Workshop on Pervasive Wireless Healthcare (MobileHealth '15), 2015, pp. 23-28.

[35] Q. Tan, W. An, Y. Han, Y. Liu, S. Ci, F.- M. Shao, and H. Tang, "Energy collecting mindful geography control with power transformation in remote sensor organizations," Ad Hoc Networks, vol. 27, pp. 44 - 56, 2015.

[36] T. He, K. W. Jawline, and S. Soh, "On remote force move and max stream in battery-powered remote sensor organizations," vol. 4, pp. 4155-4167, Aug 2016.

[37] N. Michelusi and M. Zorzi, "Optimal adaptive random multi-access in energy harvesting wireless sensor networks," IEEE Trans. Commun., vol. 63, no. 4, pp. 1355-1372, Apr 2015.

[38]D. Estrin, R. Govindan, J. Heidemann, and S. Kumar. "Next Century Challenges: Scalable Coordination inWireless Networks". In Proceedings of the fifth Annual ACM/IEEE International Gathering on Mobile Computing and Networking ( MOBICOM), pages 263-270, 1999.

[39] W. Heinzelman, A. Chandrakasan, and H. Balakrishnan. "Energy-Efficient Communication Protocols for Wireless Microsensor Networks". In Proceedings of Hawaiian International Gathering on Systems Science, January 2000.

[40]C. Intanagonwiwat, R. Govindan, and D. Estrin. "Coordinated Dissemination: A Scalable and Robust Communication Worldview for Sensor Networks ". In Proceedings of the sixth Annual ACM/IEEE International 
Conference on Mobile Figuring and Networking(MOBICOM), pages 56-67, August 2000.

[41] M. Jiang, J. Li, and Y. C. Tay. "Bunch Based Routing Protocol". Web Draft, 1999.

[42] Josef Börcsök.et.al, "Safe Position Detection Based on Safety System-on-Chip (SSoC) for Wireless IoT Application", INTERNATIONAL JOURNAL OF CIRCUITS, SYSTEMS AND SIGNAL PROCESSING, DOI: $10.46300 / 9106.2020 .14 .132, \quad$ ISSN: 19984464, Volume 14, 2020.

[43] Michael H.et.al, "Investigation of a Safety Parameter Observer for Wireless Communication", INTERNATIONAL JOURNAL OF CIRCUITS, SYSTEMS AND SIGNAL PROCESSING, DOI: 10.46300/9106.2020.14.128, ISSN: 1998-4464, Volume 14, 2020.

[44] Aymen Zreikat, "Performance Evaluation of 5G/WiFi-6 Coexistence", INTERNATIONAL JOURNAL OF CIRCUITS, SYSTEMS AND SIGNAL PROCESSING, DOI: 10.46300/9106.2020.14.116, ISSN: 1998-4464, Volume 14, 2020 